\title{
Respuestas a la migración venezolana en Bogotá: una aproximación subnacional al fenómeno
}

Responses to Venezuelan Migration in Bogotá: A Sub-National Approach to the Phenomenon

Respostas à migração venezuelana em Bogotá: uma abordagem subnacional do fenômeno

\author{
FeLIPE JARAMILLO* \\ CÉSAR NIÑO**
}

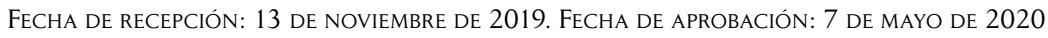

Doi: https://doi.org/10.12804/revistas.urosario.edu.co/sociojuridicos/a.8449

Para citar este artículo: Jaramillo, F., E Niño, C. (2021). Respuestas a la migración venezolana en Bogotá: una aproximación subnacional al fenómeno. Revista de Estudios Sociojurídicos, 23(1), 403-429. https://doi.org/10.12804/ revistas.urosario.edu.co/sociojuridicos/a.8449

\section{RESUMEN}

Como resultado de un proceso de investigación, el artículo analiza cómo se han construido políticas subnacionales para responder al fenómeno migratorio en Bogotá. El estudio contribuye a entender cómo las autoridades locales adoptan políticas para atender los flujos migratorios y cómo estas respuestas institucionales afectan la situación migratoria en la ciudad. Así, se argumenta que la Alcaldía Mayor de Bogotá ha adoptado un modelo liberal de gestión migratoria que propende por garantizar los derechos de los migrantes venezolanos, pero, a la vez, insiste en la importancia de

* Universidad Sergio Arboleda. Doctor en ciencia política y relaciones internacionales en el Graduate Institute of International and Development Studies. Magíster en democracia y transformaciones globales de la Universidad de Helsinki y en derecho internacional de la Universidad de los Andes. Profesor e investigador de la Escuela de Política y Relaciones Internacionales de la Universidad Sergio Arboleda (Colombia). Correo electrónico: felipe. jaramillo@usa.edu.co. ORCID: https://orcid.org/0000-0003-3766-3484

** Universidad Sergio Arboleda. Doctor en derecho internacional de la universidad Alfonso X el Sabio (España), Magíster en seguridad y defensa de la Escuela Superior de Guerra (Colombia) y profesional en política y relaciones internacionales de la Universidad Sergio Arboleda (Colombia). Actualmente, es profesor asociado de la Escuela de Política y Relaciones Internacionales de la Universidad Sergio Arboleda (Colombia). Correo electrónico: cesar.nino@usa.edu.co. ORCID: https://orcid.org/0000-0002-1417-6643 
regular su estatus migratorio. En este sentido, la investigación contribuye al estudio de las repuestas gubernamentales a los fenómenos migratorios.

Palabras clave: subnacional; migración; Colombia; políticas migratorias; derechos humanos.

\begin{abstract}
This article analyzes how the Bogota mayor's office has responded to the migration phenomenon from a sub-national perspective. The study helps to understand how local authorities adopt policies to address migratory flows and explains how these institutional responses affect the situation of migrants in the city. Thus, the research shows that the Bogota mayor's office has taken a liberal model of migration management that defends the rights of Venezuelan migrants but, at the same time, insists on the importance of regulating their immigration status. In this sense, the research contributes to the study of governmental approaches to migration flows.
\end{abstract}

Keywords: Sub-national; migration; Colombia; Bogotá; migration policies; human rights.

\title{
RESUMO
}

Como resultado de um processo de pesquisa, o artigo analisa como as políticas subnacionais foram construídas para responder ao fenômeno migratório em Bogotá. O estudo contribui para compreender como as autoridades locais adotam políticas de atendimento aos fluxos migratórios e como essas respostas institucionais afetam a situação migratória na cidade. Assim, argumenta-se que a Prefeitura de Bogotá tem adotado um modelo liberal de gestão migratória que visa garantir os direitos dos migrantes venezuelanos, mas, ao mesmo tempo, insiste na importância de regularizar sua situação migratória. Nesse sentido, a pesquisa contribui para o estudo das respostas governamentais aos fenômenos migratórios.

Palavras-chave: subnacional; migração; Colômbia; políticas migratórias; direitos humanos. 


\section{Introducción}

Diversos académicos han examinado el origen, la creación y la evolución de las políticas migratorias. Un grupo de autores estudia la regulación supranacional que se impulsó en la década de 1980 (Guiraudon, 2002; Lavenex, 2018). Otros académicos analizan la incidencia de la migración en ciertas problemáticas como el tráfico de personas, el narcotráfico, la salud pública y el crimen organizado (Ansell \& Young, 2004; Butcher \& Morrison, 1998; Karyotis, 2007). Así mismo, enfoques diversos en materia académica sobre la migración latinoamericana estudian los flujos migratorios de esta región hacia Europa. En particular, hacen énfasis en los factores que impulsan la migración como los ingresos relativos, los choques económicos y la estructura demográfica (Prieto Rosas \& López Gay, 2015). Aunque la mayoría de estas aproximaciones adoptan un enfoque Estado-céntrico, en la última década, una serie de investigaciones han propuesto visiones subnacionales al análisis de la migración (Dietz, 2010; Paquet, 2019; Smith, 2017).

El presente estudio incorpora una aproximación crítica a la creación de respuestas gubernamentales en materia migratoria desde el enfoque subnacional. A diferencia de los enfoques Estado-céntricos, que se concentran en las políticas del gobierno central, este tipo de aproximaciones explora cómo los gobiernos locales, como alcaldías y gobernaciones, responden a fenómenos transnacionales. Particularmente, en este artículo, se analizan las principales políticas, programas y proyectos de la Alcaldía Mayor de Bogotá dirigidos a atender a los migrantes.

Bogotá fue una de las primeras ciudades en América Latina y la primera en Colombia en expedir una política pública para responder al creciente flujo migratorio de venezolanos (Estupiñán, 2018). En este sentido, el análisis de las respuestas de la Alcaldía Mayor de Bogotá a la migración de venezolanos permite estudiar cómo desde las ciudades se formulan políticas de gestión migratoria. Además, al centrarse en las características de la inmigración hacia Colombia, se diferencia de los estudios más recurrentes sobre este país, los cuales han hecho hincapié en la emigración de colombianos (Bahar et al., 2018; Guarnizo, 2006; Tatar, 2019). En este orden de ideas, el estudio busca responder la siguiente pregunta de investigación: ¿cómo la Alcaldía Mayor de Bogotá 
ha construido respuestas institucionales para atender el fenómeno migratorio? Como respuesta, se argumenta que las políticas, programas y proyectos de la Alcaldía Mayor de Bogotá han propendido por un modelo liberal que reconoce los derechos de los migrantes, pero insiste en la regularización de su estatus migratorio. Al enfocarse en la formalización burocrática del inmigrante, se pasa por alto la situación de migrantes colombo-venezolanos que, aunque cumplen con los requisitos de regularización, requieren apoyos institucionales adicionales.

Para examinar cómo esta entidad ha respondido al fenómeno migratorio, se realizó una búsqueda en archivos y documentación oficial. Además, se entrevistó al personal técnico de la Secretaría de Integración Social de la Alcaldía Mayor de Bogotá, quienes son los encargados de la atención al migrante, y se radicó un derecho de petición ante esta entidad. La información recopilada se analizó a partir de los diferentes modelos de gestión migratoria que se encuentran en la literatura académica y con base en las teorías de empuje (push) y atracción (pull). Estas aproximaciones describen el tipo de relación que se da entre los migrantes y las instituciones del país de destino y sirven para identificar los factores que inciden en la permanencia o desplazamiento de las poblaciones migrantes de un lugar a otro (Castles et al., 2014). Así, como un conjunto de supuestos básicos, los modelos son una herramienta útil que permite guiar el análisis empírico (Greenwood, 1985). En definitiva, los hallazgos empíricos y las aproximaciones teóricas sobre la gestión migratoria permitieron comprender el marco regulatorio e institucional que la Alcaldía Mayor de Bogotá ha desarrollado como respuesta a la ola migratoria de venezolanos.

Así mismo, es importante aclarar que, para examinar las respuestas institucionales, no solo se tuvo en cuenta lo que está escrito, también se consideraron los silencios. Es decir, se identificaron los vacíos de política pública en materia de migración. Como señala Annica Kronsell (2006), el estudio del silencio permite entender las dinámicas y estructuras discursivas que regulan las respuestas institucionales. En este sentido, la ausencia de ciertos programas, políticas e iniciativas también debe ser analizada a partir de los modelos de gestión migratoria, en tanto que también es un reflejo de la lógica institucional para atender dicho fenómeno. Es decir, la inexistencia de respuestas institucionales condiciona la 
relación que se pretende entablar entre los migrantes y las instituciones de la ciudad de recepción. Por esta razón, la ignorancia institucional no puede ser desatendida al momento de examinar cómo las autoridades locales atienden el fenómeno migratorio (Paul \& Haddad, 2019).

El artículo está dividido en cuatro secciones. En primer lugar, se presenta el debate en la literatura sobre la regulación internacional de la migración y los derechos humanos. Así mismo, se discuten los diferentes modelos de gestión migratoria. En seguida, se aborda el enfoque subnacional como una aproximación que nutre la discusión sobre los flujos migratorios a nivel local y los modelos de gestión migratoria. Particularmente, se presentan las teorías de empuje y atracción que se encuentran en la literatura. En tercer lugar, se analiza la inmigración en Colombia y, posteriormente, se presentan los hallazgos sobre cómo la Alcaldía Mayor de Bogotá ha respondido ante las presiones migratorias. Finalmente, en las conclusiones, se destaca cómo el caso de Bogotá, producto de la investigación, brinda una nueva perspectiva al análisis de los fenómenos migratorios en América Latina y el mundo.

\section{Los modelos de gestión migratoria}

Uno de los debates académicos en torno al fenómeno de la migración es la discusión sobre la tensión entre las aproximaciones que hacen hincapié en la protección de los derechos humanos de los migrantes y aquellas que buscan la regulación de los movimientos migratorios (Crépeau \& Nakache, 2006). La literatura sobre movimientos migratorios forzados tiende a evidenciar cómo estas dos aproximaciones se construyen bajo lógicas antagónicas (Aleinikoff, 2003). Es decir, los derechos humanos se conciben como un obstáculo para la regulación de los flujos migratorios y, viceversa, el control de los flujos migratorios se entiende como una barrera para la protección de los derechos humanos.

El interés nacional es el zócalo que legitima la administración de los flujos migratorios (Bade, 2004). Desde esta perspectiva, los Estados cuentan con la autoridad para controlar y regular el movimiento de personas a través de las fronteras y propenden por maximizar sus intereses económicos y de seguridad (Crépeau \& Nakache, 2006). 
En otras palabras, los Estados formulan políticas de admisión, residencia, expulsión y naturalización para no-ciudadanos conforme a sus propios intereses. Esta administración de las fronteras produce diferentes categorizaciones de migrantes.

Las prerrogativas y derechos de los migrantes dependen de su categorización (Goodwin-Gill, 2007). Por ejemplo, a los migrantes irregulares se les expulsa, a los migrantes regulares se les permite residir en el territorio. Sin embargo, según Bade (2004), esta categorización contribuye a la persistencia de la imagen del fenómeno migratorio como una amenaza a la seguridad nacional. Así, la migración ha pasado a ser uno de los principales temas de la agenda internacional, en tanto que los Estados desean garantizar la seguridad e integridad de sus fronteras (Adamson, 2006).

El incremento de la migración nutre los discursos de riesgo, las formas modernas de racismo y los nuevos métodos de securitización (Ibrahim, 2005). Así, para François Crépeau et al. (2007), la prevalencia de las políticas migratorias relacionadas con la seguridad nacional ha generado una percepción negativa del extranjero, especialmente del migrante irregular. Como resultado, se asocia al migrante con la ilegalidad y criminalidad. En la práctica, se ha visto un deterioro de los derechos políticos y civiles básicos de los migrantes y un incremento de obstáculos que limitan el acceso de estos a los derechos económicos y sociales, incluido el derecho a la salud (Goodwin-Gill, 2008).

Cada vez más, los migrantes se convierten en chivos expiatorios de los problemas sociales, como la reducción de salarios, los problemas económicos, el incremento de violencia y la ineficiencia de los servicios públicos (Düvell, 2006). El aumento de los estereotipos fortalece el discurso de la amenaza migratoria (Léonard, 2010). Así, se incita al público a pedir políticas de gestión migratoria más estrictas que limiten los movimientos fronterizos y combatan la migración irregular (Goodwin-Gill, 2007). En tal sentido, se configura una interacción simbiótica entre la agencia del migrante y su relación con la comunidad receptora (Paquet, 2019; Tatar, 2019). En otras palabras, como señala Everett Lee (1966), la relación entre corrientes y contracorrientes migratorias depende de que "la migración es considerada selectiva y el grado de selectividad depende de un número de factores los cuales a menudo dan como resultado una selección bimodal" (p. 47). 
Ante el crecimiento de respuestas institucionales a los movimientos poblacionales transfronterizos, un grupo de académicos ha desarrollado proposiciones de tipo teórico que buscan caracterizar las políticas púbicas de los Estados receptores dirigidas a la atención de los inmigrantes (Castles et al., 2014). Para estos académicos, los modelos son una herramienta valiosa para explorar cómo los Estados receptores intentan equilibrar la obligación de respetar los derechos humanos y, al mismo tiempo, atender los desafíos a nivel jurídico-político que representa el fenómeno migratorio (Navarrete-Yáñez, 2017). En términos generales, los modelos son una forma de comprender la gestión de los Estados receptores frente a los inmigrantes, que se entiende como el conjunto de políticas públicas generales y sectoriales orientadas a definir los parámetros de admisión, residencia, expulsión y naturalización para no-ciudadanos (Newton, 2008).

En la literatura existe una diversidad de marcos de análisis para definir los modelos de gestión migratoria (Navarrete-Yáñez, 2017). Por ejemplo, un primer marco de análisis diferencia los modelos migratorios a partir del tipo de interacción entre las instituciones del Estado y los migrantes. La premisa básica es que la integración de la población inmigrante depende de las estructuras institucionales que regulan la relación entre el individuo, el Estado y la sociedad. Bajo este marco de ideas, Soysal y Soyland (1994) identifican cuatro tipos de modelos: corporativista, liberal, estadista y fragmentado. En el modelo corporativista, los gobiernos generan políticas verticales que buscan integrar a los inmigrantes de manera colectiva. Por consiguiente, la responsabilidad de integrar a los migrantes recae sobre el Estado. Este debe brindar mecanismos institucionales para que los inmigrantes puedan relacionarse con los órganos públicos de manera similar a cualquier otra colectividad corporativizada, en tanto que se los vincula a la noción de minorías étnicas. Por el contrario, en el modelo estadista, se administra la migración de forma centralizada y el Estado no considera a los inmigrantes como un colectivo sino como un conjunto de individuos, en otras palabras, se les configura de manera particularizada para el acceso a servicios y materialización de políticas públicas. Así, el Estado define líneas de acción sobre los servicios públicos a los que los migrantes tienen acceso y las actividades en las que pueden participar. Por otro lado, el 
modelo liberal posiciona al individuo como una fuente de autoridad y legitimidad. Como este modelo se caracteriza por la descentralización, la incorporación se da principalmente a través de asociaciones voluntarias que asumen la función de apoyar a los inmigrantes y el eje principal de la integración se da por medio del mercado laboral. Por último, el modelo fragmentado es una mezcla del modelo corporativista y estadista. En este sentido, la incorporación del migrante es parcial y no depende del Estado sino de organizaciones sociales como gremios laborales o religiosos (Soysal \& Soyland, 1994).

Otro marco analítico para comprender los modelos de gestión migratoria se enfoca en los criterios para acceder a los beneficios de la ciudadanía. Al desarrollar este marco analítico, Stephen Castles et al. (2014) identifican tres modelos predominantes: el modelo multicultural, el de asimilación y el étnico o de exclusión diferencial. El modelo multicultural reconoce que el principio de la integración requiere una mutua acomodación tanto por parte de la población inmigrante, como de la sociedad receptora. Así, no se espera que renuncien a su propia cultura, religión e idioma. Sin embargo, sí existe una expectativa de que deben acoplarse a ciertos valores de la sociedad receptora. En el modelo de asimilación, los inmigrantes deben adoptar la cultura (modos e identidades) de la sociedad receptora para acceder a los beneficios de la ciudadanía. Esto implica que el inmigrante debe abandonar sus tradiciones culturales, idioma y creencias. Por el contrario, el modelo étnico o de exclusión se fundamenta en reglas discriminatorias de inmigración. Por esta razón, se limita el acceso a la ciudadanía a partir de criterios étnicos o raciales, motivo por el cual se puede segregar a la población en cuanto al acceso a los beneficios de la ciudadanía. Aunque se permite que el inmigrante acceda a ciertos espacios, como el mercado laboral, se le impide que participe activamente en la administración política de la comunidad.

Emily R. Gill (2016) propone un marco analítico que se centra en la existencia o ausencia de consentimiento entre la comunidad receptora y los inmigrantes. Con base en este criterio, la autora identifica dos tipos de modelos de ciudadanía: el modelo adscriptivo y el modelo consensual. Por un lado, en el modelo adscriptivo, el inmigrante puede demandar acceso a los beneficios de la ciudadanía siempre y cuando 
cumpla con ciertas características y no requiere el consentimiento de la comunidad receptora. Bajo este modelo, la ciudadanía es un derecho que se da por nacimiento, ya sea por ius soli, nacimiento en el territorio, o ius sanguinis, por un vínculo sanguíneo con un ciudadano. Por el contrario, el modelo consensual rechaza el derecho a los beneficios de la ciudadanía por nacimiento y favorece políticas de acceso que se basen en consenso. Como consecuencia, la membresía de facto en la comunidad a través de los lazos sociales es lo que otorga a los individuos el estatus de ciudadanos.

Ahora bien, a pesar de las diferencias, los modelos de gestión migratoria se centran en la potestad del Estado de admisión, residencia, expulsión y naturalización para no-ciudadanos conforme a sus propios intereses. Esto hace que la mayoría de las aproximaciones adopten un enfoque Estado-céntrico que se concentra en las políticas de gestión migratoria de los gobiernos centrales. Como los gobiernos locales (las alcaldías y las gobernaciones) carecen de estos poderes, no se suele hacer hincapié en las respuestas institucionales a la migración que se dan a nivel subnacional. ${ }^{1}$ Sin embargo, en el siguiente apartado, se discute la importancia de pensar modelos subnacionales para analizar cómo las ciudades entablan la relación con los inmigrantes. Para desarrollar esta propuesta teórica, se debe partir del presupuesto de que si bien las autoridades locales no cuentan con la potestad de definir los criterios de admisión y expulsión, sí son un actor clave en el entendimiento de las respuestas institucionales a los flujos migratorios.

\section{Aproximaciones críticas de lo subnacional en las migraciones}

Las relaciones internacionales contemporáneas se enfrentan a un cúmulo dinámico de fenómenos que trascienden los clásicos modelos Estado-céntricos. Los asuntos en la agenda han empezado a sufrir

Las respuestas subnacionales dependen de las características del Estado receptor. Por ejemplo, en el caso suizo, la respuesta institucional depende del cantón (Bennour $\mathcal{E}$ Manatschal, 2019). 
procesos de involucramiento mayor a escalas subnacionales que comprometen en buena medida las formas de construir los procesos de toma de decisiones desde una perspectiva bottom-up. Es decir, cada vez más, la formulación de políticas acoge un sistema ascendente, en el cual los marcos regulatorios se construyen de abajo hacia arriba.

En efecto, las clásicas lógicas de entendimiento fenomenológico desde los procesos exclusivamente nacionales, lograron opacar las realidades locales y ocultaron las dinámicas estructurales de las configuraciones regionales (Suárez-Cao et al., 2017). En ese sentido, los trabajos de Suárez-Cao et al. (2017) advierten que ha habido una desatención sistemática sobre la política local y focalizada en América Latina; no obstante, la apuesta por comprender las cuestiones domésticas con influencia nacional han tenido un gran acervo en las propuestas metodológicas de Giraudy et al. (2019). Aquella perspectiva demuestra cómo la investigación subnacional proporciona información útil sobre temas sustantivos disciplinares desde los regímenes y la representación, hasta Estados y seguridad, incluyendo el desarrollo social, económico y el fenómeno migratorio (Giraudy et al., 2019).

De tal manera, la necesidad de establecer lecturas territoriales, incluso desde nociones particulares, representa una segmentación importante para la investigación causal, evitando clasificaciones homogeneizadoras y universalistas que redundan en arquetipos tradicionales. Para autores como Schiavon (2015), la creciente globalización e interdependencia del sistema internacional ha disminuido los costos de participación de nuevos actores en las relaciones internacionales $\mathrm{y}$, como inferencia causal, ha llevado a una proliferación de instituciones y temas que deben ser tratados en la agenda internacional desde una perspectiva local, y desde un enfoque "glocal". Este último término, alcanza una construcción ecléctica y holística que despoja las narrativas Estado-céntricas y evoca visiones complejas que no hacen parte de una elección sobre la cosmovisión del territorio (Murga-Menoyo \& Novo, 2017), es entonces un concepto articulado que representa las afectaciones globales y locales en sinergia con una resignificación de lo doméstico y lo internacional.

Para autores como Duchacek (1984), la cuestión subnacional en la escena internacional se ha convertido en un hecho del mundo interdependiente que cohabita en conflicto con la política exterior nacional. 
De estas actividades surge el concepto de un estado territorial como actor multivocal, es un efecto de la interdependencia contemporánea (Duchacek, 1984; Hurrell, 2013; Global South Studies Center, 2015).

Con base en lo anterior, la interdependencia compleja (Keohane $\mathcal{E}$ Nye, 1977; Nye, 2010), así como los problemas domésticos han alentado a muchos gobiernos locales a ampliar sus competencias focalizadas a internacionales. En esencia, han buscado replantear la brecha entre el fenómeno, la sensibilidad y la vulnerabilidad en asuntos relacionados con sus respectivas normas y competencias jurídicas, así como la promoción de temas referentes al comercio, la inversión extranjera, los derechos humanos y cuestiones medioambientales, de turismo y de migración (Duchacek, 1984). Este último se ha convertido en un asunto que ha moldeado las visiones tradicionalistas sobre las relaciones internacionales de una aproximación realista, estática y de exclusiva relación al interés nacional (Kolodziej, 2005; Morgenthau, 1949) a una dinámica con una amplia agenda de cuestiones estructurales de la gobernanza global y de la difuminada noción física de fronteras (Betts \& Kainz, 2017); de hecho se convierte en un referente fenomenológico de análisis sobre flujos y contraflujos e implicaciones en las políticas públicas subnacionales.

A saber, para comprender desde una lógica subnacional las políticas en clave de la migración, es imperante tener puntos conceptuales que funcionen como temas causales en la adopción de marcos normativos y en el devenir instrumental de códigos y regímenes. Para Betts y Kainz (2017), la gobernanza global de la migración se puede definir como el conjunto de normas y estructuras organizativas que regulan las respuestas institucionales al fenómeno de la migración. En consecuencia, los Estados trabajan colectivamente de manera que puedan cumplir con las amplias demandas en las interconexiones transfronterizas; adicionalmente, ha habido un rápido incremento de la movilidad humana a través de las fronteras: "en 2019, el número de migrantes alcanzó la cifra de 272 millones, 51 millones más que en 2010" (Organización de las Naciones Unidas, 2019), lo que representa el $3.5 \%$ de la población mundial (Betts \& Kainz, 2017; Boswell, 2002).

Con base en lo anterior, las construcciones de los flujos migratorios han tenido un desenvolvimiento clave alrededor de los fenómenos que moldean la naturaleza migratoria; es decir, en condiciones teóricas, hay 
algunos tipos de modelos y aproximaciones que intentan leer y explicar las razones de estas. Por un lado las macroteorías que enfatizan en las condiciones estructurales objetivas denominadas de empuje (push) y los factores de atracción ( pull) enmarcadas en causas económicas, laborales, acción racional por salarios o razones vitales como la represión estatal o conflictos y violencia (Boswell, 2002). Por el otro, las mesoteorías intentan controvertir la visión anterior y se centran más en una dinámica estructural entre redes y sistema; es decir, suponen que la migración pone su cauce dentro de un sistema de migración aludiendo a que la sensibilidad e interdependencia de país puede generar un flujo más continuo de migrantes (Faist, 2000).

Empero, la literatura alrededor de la migración parece estar volcada en gran medida a las explicaciones de la movilidad humana, sus causas y las implicaciones sociales, de seguridad y económicas, entre otras, pero no posee un gran asidero empírico ni teórico sobre los diseños e instrumentos políticos de los actores subnacionales en países en vías de desarrollo como nuevos receptores de aquellos flujos. En otras palabras, la literatura se ha preocupado más por los corredores migratorios entre los países del Sur Global al Norte Global con sus efectos relacionados a la seguridad y la economía (Ayoob, 1991; 2002; Butcher \& Morrison, 1998; Sharp, 2011) que por las variables de cumplimiento y adopción subnacional de los flujos del Norte y Sur al propio Sur -en esta oportunidad a las dimensiones de políticas migratorias en Bogotá-.

Ahora bien, para explorar las respuestas institucionales subnacionales al fenómeno de la migración es necesario repensar los modelos de gestión de la migración y las teorías de empuje y atracción. Un primer paso para avanzar hacia esta reconceptualización consiste en entender los poderes que poseen las autoridades locales para atender a la población inmigrante. En este sentido, se parte del presupuesto de que los alcaldes y gobernadores no son los que definen los criterios de ingreso de los inmigrantes a su territorio y tampoco pueden determinar las reglas de expulsión. No obstante, sí pueden desarrollar políticas de gestión migratoria que afectan los factores de empuje y atracción. Por ejemplo, las autoridades locales pueden crear centros de atención para brindar a la población inmigrante información que facilite su asentamiento o incorporación al mercado laboral. Así mismo, pueden implementar políticas 
que propendan por el intercambio cultural o programas que busquen la integración a la sociedad. De tal manera, en el caso de la ciudad de Bogotá, los determinantes para la atracción de los migrantes tienen una relación especial con los canales y dispositivos de atención sumados a los espacios de oportunidad compleja en el territorio. En efecto, se sincronizan las motivaciones personales del migrante para escoger a Bogotá sobre otras regiones de Colombia con condicionantes de acceso a recursos y movilidad humana (Adamson, 2006; Betts \& Kainz, 2017).

La reconfiguración de los marcos analíticos es el segundo paso hacia la formulación de modelos teóricos que sirvan para comprender las respuestas subnacionales. Por consiguiente, se debe definir si la construcción de los modelos de gestión migratoria se fundamenta en el consentimiento, relación institucional, criterios de aceptabilidad u otros factores. Adicionalmente, es importante comprender si los modelos de gestión migratoria subnacionales tienden a privilegiar programas multiculturales, de asimilación o de exclusión. En suma, los proyectos investigativos que analicen la gestión de la migración desde un nivel subnacional deben partir del presupuesto de que las autoridades locales también pueden incidir en los factores de empuje y atracción e igualmente incorporan modelos de gestión para responder al flujo de inmigrantes.

\section{El fenómeno de la migración en Colombia}

Colombia ha tenido una importante historia de emigración en el siglo $\mathrm{XX}$, marcada por la violencia y el conflicto armado interno (Banco Mundial, 2018; Tovar, 2006). Incluso, Venezuela era un destino tradicional de los ciudadanos colombianos que decidían emigrar por múltiples causas, entre ellas la violencia en el país. En efecto, Colombia no posee experiencias sobre recepciones de grandes flujos dinámicos de migrantes al territorio y las instituciones nacionales no tienen diseños técnicos ni metodológicos lo suficientemente robustos para las lógicas contemporáneas.

En materia de migración, Colombia ha tenido un desacierto en la comprensión del fenómeno; es decir, las configuraciones técnicas e instrumentos jurídicos concernientes al flujo migratorio se han concentrado en mayor medida en temas fronterizos por vía terrestre (Ministerio de 
Relaciones Exteriores de Colombia, 2003). Partiendo de lo anterior, Colombia entiende casi de manera exclusiva que el ingreso de personas al territorio nacional tiene una vinculación directa con las fronteras físicas tradicionales. En ese sentido, el entendimiento fragmentado que la política nacional posee alrededor del asunto es que, en materia territorial, los migrantes de fronteras vecinas llegan al lado colombiano a entidades territoriales próximas. En otras palabras, la arquitectura nacional contempla que personas provenientes de Venezuela habitan en Colombia regiones como La Guajira, Cesar, Norte de Santander, Boyacá, Arauca, Vichada y Guainía; los provenientes de Ecuador en Nariño y Putumayo; los de Panamá, Chocó, y los de Perú y Brasil en Putumayo, Amazonas y Vaupés (Ministerio de Relaciones Exteriores de Colombia, 2011). Lo anterior es un reflejo de desconexión, desconocimiento y fragmentación sobre el fenómeno de la migración a pesar de los intentos nacionales de atender la cuestión de una manera integral.

Las dinámicas políticas, sociales y económicas en Venezuela han generado un empuje de la migración hacia Colombia. En ese sentido, los flujos migratorios han impactado el proceso de toma de decisiones tanto nacionales como subnacionales, arrojando dislocaciones importantes. Mientras que por un lado las políticas nacionales en Colombia están volcadas a la atención, registro y control fronterizo por medio de las oficinas y dependencias adscritas a la Cancillería; por el otro, el flujo irregular a los territorios no fronterizos ha generado traumas institucionales que han tenido que repensar la dinámica y crear insumos de política pública para su atención.

A saber, desde el 2014 se han registrado alrededor de 1.4 millones de venezolanos en Colombia de 2.3 millones que han salido en total (Proyecto Migración Venezuela, 2019). En ese sentido, el registro en Bogotá de migrantes irregulares atendidos por el gobierno distrital provenientes de Venezuela es de aproximadamente 4360 personas; es tan solo hasta el 2018 que se crea en la ciudad el Centro Integral de Atención al Migrante -CIAM- (Secretaría Distrital de Integración Social, 2018). Con base en lo anterior, el análisis redunda en las explicaciones deductivas que surgen debido a que es el fenómeno migratorio en Colombia el que ha motivado la arquitectura de instrumentos y políticas para el migrante dentro del país. 


\section{Una aproximación a las respuestas institucionales a la migración venezolana}

Para el caso particular de Bogotá, esta es la ciudad con mayor número de migrantes (estimado entre 88 y 112 mil), que representa el $9.8 \%$ del total de personas provenientes de Venezuela (Banco Mundial, 2018). Bogotá tiene gran capacidad de absorción migrante, pero el alto número de personas irregulares traza líneas precisas sobre los desafíos institucionales que se alejan de los clásicos problemas de frontera. Por un lado, al existir una alta densidad urbana, se imponen retos para mitigar la formación de asentamientos informales $\mathrm{y}$, por el otro, la toma del espacio público para fines irregulares (Banco Mundial, 2018). No obstante, el fenómeno migratorio ha llevado a que la administración distrital haya tomado medidas pensando en el migrante venezolano, por ello, desde el CIAM se han venido desarrollando proyectos de atención sobre programas de jardines infantiles enfocados en la protección y erradicación progresiva del trabajo infantil por entidades como los Centros Amar, la atención de las comisarías de familias y los comedores comunitarios (Secretaría Distrital de Integración Social, 2018).

E1 12 de septiembre de 2018, el alcalde Enrique Peñalosa propuso una política integral de acción frente a los migrantes venezolanos, tornando a Bogotá en la primera ciudad de Colombia en articular este tipo de medidas. Al lanzar esta política, el alcalde afirmó:

Estamos creando una gerencia especial para atender a los migrantes venezolanos. Aquí estamos tomando una serie de medidas en una resolución que asigna responsabilidades a las diferentes Secretarías del Distrito. Además, creamos un registro social de migrantes para atender las personas (Libian Barreto, 2018).

Así mismo, anunció la creación de 50 alojamientos temporales humanitarios en los cuales los casos más prioritarios podrán encontrar alimentación por tres días. Por su parte, el secretario general de la Alcaldía, Raúl Buitrago, manifestó que estos programas de atención "permitirán avanzar en la normalización del estatus migratorio de los venezolanos y orientar en servicios básicos" (Libian Barreto, 2018). 
En este sentido, el secretario de gobierno, Miguel Uribe, destacó que "con estas acciones Bogotá se posiciona como la primera ciudad del país que expide, a través de un acto administrativo, una circular que contempla y articula los servicios que se prestarán ante la situación de los venezolanos" (Libian Barreto, 2018).

Al analizar estos discursos institucionales sobre el fenómeno migratorio, se evidencia que la Alcaldía Mayor de Bogotá ha adoptado un modelo liberal que reconoce los derechos de los migrantes, pero también insiste en la regularización del estatus migratorio. En este sentido, la integración es entendida como sinónimo de normalización $\mathrm{y}$, por consiguiente, el "registro social" se torna la piedra angular de la respuesta institucional. Además, el énfasis se da en el individuo. Es decir, no se busca organizar a los inmigrantes venezolanos como colectivo o como una corporación poblacional. Por el contrario, los servicios de la Alcaldía Mayor de Bogotá se enfocan en la atención individualizada del migrante.

En noviembre de 2018, ante la creciente presencia de migrantes venezolanos en Bogotá, se creó una mesa de trabajo para abordar este fenómeno y evaluar las posibles respuestas. Aquel ejercicio estuvo conformado por autoridades de orden nacional y distrital con un gran componente de agencias de cooperación internacional, grupos gremiales y la Cámara de Comercio de Bogotá (Estupiñán, 2018). Dicha mesa gestionó la formación de un cargo administrativo distrital de "gerencia para venezolanos" lo cual constituye la situación de urgencia frente al flujo de personas de dicho país. En efecto, la "gerencia para venezolanos" se encarga de administrar, atender y velar por el tránsito de irregularidad al de regularidad del migrante, también tiene funciones de atención sobre las necesidades básicas y la urgencia de satisfacción de condiciones humanitarias (Semana, 2018).

Una vez más, el análisis de las respuestas institucionales evidencia la prevalencia de un modelo de gestión migratorio que insiste en el tránsito hacia la regularidad del migrante. Así, el objetivo principal de la Alcaldía Mayor de Bogotá ha sido normalizar el estatus migratorio de los migrantes que se encuentran en la ciudad. Sin embargo, este modelo ignora a los migrantes venezolanos con nacionalidad colombiana. Como estos migrantes ya hacen parte de la regulación burocrática, se 
asume que tienen las mismas oportunidades que los colombianos y no necesitan una atención diferencial.

Como respuesta a un derecho de petición, la Secretaría de Integración Social insistió en que la competencia en materia de política migratoria recae en el Gobierno nacional. Así mismo, señaló que las acciones desarrolladas por esta secretaría se ceñían al documento Conpes 3950 de 2018. En este marco, la Alcaldía informó que la Secretaría de Integración Social presta servicios a las personas en mayor situación de pobreza y vulnerabilidad. En el caso de los migrantes, esta atención es transitoria y opera a través de dos unidades, el CIAM y el SuperCade Social de la Terminal de Transportes del Salitre. En estas unidades, los migrantes venezolanos reciben orientación, información y referenciación. También se les brinda atención psicosocial y jurídica (Respuesta a derecho de petición de los autores, 2019).

La Circular 037 de 2018 de la Secretaría de Integración Social traza las rutas de atención para la población vulnerable de origen extranjero. Esta circular establece:

En la actualidad es un hecho notorio que se presenta una fuerte migración de ciudadanos venezolanos hacia nuestro país, como resultado de la realidad social, política y económica que se está presentando en la República Bolivariana de Venezuela, siendo Bogotá una de las principales ciudades receptoras de esta población. Por la anterior, se hace necesario que la Secretaría Distrital de Integración Social tome medidas necesarias para darle cumplimiento a la normatividad constitucional, los tratados internacionales suscritos por Colombia y la jurisprudencia vigente que regula el tema de las personas extranjeras, y pueda brindar la atención debida por su condición de vulnerabilidad (Alcaldía Mayor de Bogotá, 2018).

En este sentido, se puede evidenciar que la secretaría hace un llamado al respeto de los derechos humanos de los migrantes y resalta su función como protectora y garante de los derechos de esta población vulnerable. Así, el discurso se centra en la instrumentalización legal y el cumplimiento de los estándares y obligaciones constitucionales e internacionales. 
Como lo establece la circular:

Ha de tenerse de presente que en ningún caso el legislador está habilitado para desconocer la vigencia y el alcance de los derechos fundamentales garantizados en la Constitución Política y en los Tratados internacionales en el caso de los extranjeros, así aquéllos se encuentren en condiciones de permanencia irregular en el país (Alcaldía Mayor de Bogotá, 2018).

Bajo este marco, la irregularidad es una construcción legal que no priva a la persona de sus derechos fundamentales como ser humano. Estas respuestas institucionales encajan en el modelo liberal. Desde esta aproximación, es posible argumentar que el eje de la política de gestión migratoria de la Alcaldía Mayor de Bogotá se ha centrado en el establecimiento de medidas que prohíban la discriminación individual de los migrantes. Por lo tanto, el llamado a las entidades del distrito ha sido que no discriminen a los migrantes venezolanos y atiendan aquellas poblaciones que enfrentan situaciones de crisis humanitaria. En términos generales, la lógica institucional vela para que los servicios de las entidades de la Alcaldía Mayor de Bogotá para la población en general beneficien a los migrantes venezolanos.

Adicionalmente, del análisis de las circulares se desprende que el dilema entre la seguridad y la dignidad humana se matiza a nivel subnacional. En otras palabras, prevalece la protección y la obligación de proteger al migrante y no proteger(se) del migrante, lo que puede comprenderse como un proceso de desecuritización del fenómeno migratorio. En suma, para la Secretaría de Integración Social:

Las autoridades colombianas no pueden desatender el deber de garantizar la vigencia y el respeto de los derechos constitucionales de los extranjeros y de sus hijos menores de edad. La ley no puede restringir, en razón de la nacionalidad, los derechos reconocidos en la Constitución y en los tratados y convenios internacionales sobre derechos humanos, dado que ellos son inherentes a la persona y tienen un carácter universal (Alcaldía Mayor de Bogotá, 2018). 
Con el fin de darle alcance a la Circular 037 del 24 de diciembre de 2018, la Secretaría de Integración Social emite la Circular 012 del 2 de abril de 2019. En ella, se centra en la situación de los niños migrantes y se precisa que "no habrá ninguna restricción, incluso si sus padres o acudientes se encuentran en una situación irregular en el país, para el acceso a los servicios de educación inicial brindados por esta Secretaría" (Alcaldía Mayor de Bogotá, 2019). Por consiguiente, se adopta el modelo liberal y se hace hincapié en la necesidad de proteger los derechos de los migrantes independientemente de su categorización.

En la entrevista entre los autores y los funcionarios de la Secretaría de Integración Social, se conversó sobre la imagen que a nivel distrital se ha esbozado sobre el migrante venezolano. En efecto, las dinámicas institucionales se han convertido en catalizadores que reaccionan a los problemas domésticos y se construyen dispositivos para la regulación del individuo. Empero, la arquitectura jurídica e instrumental sobre la atención al migrante refleja que el fenómeno irregular ha construido las respuestas y alcanza a determinarse como un factor entrópico que desborda las capacidades institucionales para la atención.

Con base en lo anterior, la protección de los derechos humanos no implica el abandono de las preocupaciones sobre la seguridad nacional y ciudadana. Por el contrario, los funcionarios expresaron que las políticas y programas de asimilación del migrante son la mejor herramienta para evitar que el fenómeno migratorio se securitice.

Para quebrar las barreras sociales y los sentimientos de xenofobia, el distrito lanzó las iniciativas Hinchas sin Fronteras, Somos Panas y Venezuela Aporta. Estos tres programas buscan integrar a la población venezolana a las actividades de la ciudad, reducir los niveles de xenofobia e intolerancia y estimular actividades culturales, gastronómicas y de emprendimiento de los migrantes en Bogotá. En términos de cifras de atención subnacional, 1694 niños y niñas migrantes están matriculados en los jardines infantiles diurnos, 302 personas en comedores comunitarios y 1318 en enlace social. Además, el CIAM ha beneficiado a 4360 migrantes (Estupiñán, 2018).

Estos esfuerzos institucionales para evitar la discriminación también encajan bajo el modelo liberal. En este sentido, lo que se busca es que 
sea más fácil para los migrantes venezolanos integrarse a la sociedad colombiana. Estos programas no buscan formar colectivos u organizaciones de venezolanos que articulen sus demandas ante las instituciones locales, sino promover lógicas de inclusión individual que permitan que el inmigrante venezolano se acople y sea acogido por los residentes de la capital.

Ahora bien, un ejemplo de cómo las autoridades locales inciden en las dinámicas de empuje y atracción es la creación del albergue en el barrio Luisa María Fernández. Esta iniciativa la formuló la Alcaldía Mayor de Bogotá con el fin de tratar los asentamientos irregulares de los migrantes venezolanos en la ciudad, situación que produjo conflictos sociales con los residentes del sector en la localidad de Engativá (E1 Tiempo, 2018a). En ese sentido, la materialización de recursos físicos y la asignación de un espacio geográfico como enclave urbano de la migración (Capron \& Esquivel, 2016) repercutió en dimensiones de percepción sobre la inseguridad y la violencia en ese sector de la ciudad (Estupiñán, 2018). Sin embargo, la administración distrital construyó un manual de convivencia y comportamiento específico para el albergue, un código de comportamiento que incluye horarios de entrada y salida al enclave, derechos y deberes, cuestiones sobre la prohibición de venta de sustancias alucinógenas, porte de armas, limpieza del lugar, etc.; el incumplimiento de estas reglas de juego podría incluso llevar a la deportación (E1 Tiempo, 2018b).

Los albergues fueron un instrumento para normalizar a los inmigrantes y securitizar los asentamientos irregulares de los migrantes venezolanos en la ciudad. A los migrantes se les permitió regularizar su estatus migratorio. A aquellos que rechazaron esta oportunidad se les ofreció la posibilidad de regresar a su país natal o de tomar un rumbo diferente (Zapata, 2019). Además, la Alcaldía Mayor de Bogotá se comprometió a proveer transporte para aquellos migrantes que decidieran no permanecer en la ciudad.

Estos esfuerzos para normalizar y securitizar el flujo migratorio contribuyeron a la construcción de clasificaciones sociales de los migrantes irregulares como el "otro" (Mouffe E Laclau, 2007). En efecto, la iniciativa tornó el asunto de los migrantes venezolanos irregulares en un problema clásico de seguridad urbana, motivo por el que el 
campamento humanitario fue desmontado (Zapata, 2019). En suma, la Alcaldía Mayor de Bogotá participó en los factores de empuje y atracción, insistiendo en la regularización de los inmigrantes venezolanos que desearan permanecer en la ciudad, "ofreciendo" alternativas de salida para aquellos que no normalizaran su estatus migratorio.

\section{Conclusiones}

La presente investigación permitió hacer un rastreo de instrumentos de política pública en materia de migración en Bogotá. En efecto, se encontró que las políticas subnacionales para responder al fenómeno migratorio en la ciudad han estado enmarcadas en el flujo de migrantes venezolanos. En ese sentido, las respuestas institucionales fueron forjadas gracias a la migración venezolana que motivó la creación del CIAM.

Con base en lo anterior, las medidas implementadas por la administración distrital adoptan un modelo liberal de la gestión migratoria. En este sentido, aunque se vela por la no-discriminación y la protección de los derechos humanos, el objetivo principal es normalizar el estatus migratorio de los venezolanos que residen en la ciudad. Así mismo, la atención a los migrantes por parte de las entidades de la Alcaldía Mayor de Bogotá se fundamenta en el individuo. Es decir, no se busca articular demandas colectivas de los migrantes venezolanos, sino brindar atención individualizada que permita la asimilación a la sociedad.

Esta aproximación liberal, sin embargo, pasa por alto la situación de los migrantes venezolanos que tienen nacionalidad colombiana. Bajo la lógica liberal, esta población ya cuenta con condiciones de "igualdad" ante la ley y, por consiguiente, tiene las mismas oportunidades que los nacionales colombianos. Ante esta situación, se evidencia que la prevalencia del modelo liberal ignora las condiciones estructurales que impiden que la igualdad formal se materialice en una igualdad efectiva.

Por último, el análisis de las políticas de gestión migratoria permite romper el paradigma Estado-céntrico que ignora las respuestas de las autoridades subnacionales a fenómenos transnacionales. Como conclusión, el estudio permite encontrar que estas entidades locales participan en la gestión del fenómeno migratorio, en tanto que incorporan modelos de 
respuesta y son un actor clave para la construcción de las condiciones de empuje y atracción de la población migrante.

\section{Referencias}

Adamson, F. B. (2006). Crossing borders: International migration and national security. International Security, 31(1), 165-199. https://doi.org/10.1162/ isec.2006.31.1.165

Aleinikoff, T. A. (2003). International legal norms and migration: a report. En T. Alexander Aleinikoff \& Vincent Chetail (Eds.), Migration and international legal norms (Vol. 2) (pp. 123-136). TMC Asser Press.

Ansell, N., E Young, L. (2004). Enabling households to support successful migration of AIDS orphans in southern Africa. AIDS Care, 16(1), 3-10. https:// doi.org/10.1080/09540120310001633921

Ayoob, M. (1991). The security problematic of the third world. World Politics, 43(02), 257-283. https://doi.org/10.4324/9781315184517-15

Ayoob, M. (2002). Inequality and theorizing in international relations: The case for subaltern realism. International Studies Review, 4(3), 27-48. https:// doi.org/10.1111/1521-9488.00263

Bade, K. (2004). Legal and illegal immigration into Europe: Experiences and challenges. European Review, 12(3), 339-375. https://doi.org/10.1017/ S1062798704000316

Bahar, D., Dooley, M., \& Huang, C. (2018). Integrating Venezuelans into the Colombian labor market: Mitigating costs and maximizing benefits. Brookings Global Economy and Development. https://reliefweb.int/sites/reliefweb. int/files/resources/Integrating $\% 20$ Venezuelans $\% 20$ into $\% 20$ the $\% 20$ Colombian $\% 20$ labor $\% 20$ market $\% 20-\% 20$ Mitigating $\% 20$ costs $\% 20$ and $\% 20$ maximizing $\% 20$ benefits.pdf

Banco Mundial. (2018). Migración desde Venezuela a Colombia: impactos $y$ estrategia de respuesta en el corto y mediano plazo. https://openknowledge.worldbank.org/bitstream/handle/10986/30651/131472SP. pdf?sequence $=3 \&$ isAllowed $=y$

Bennour, S., \& Manatschal, A. (2019). Immigrants' feelings of attachment to Switzerland: Does the Cantonal context matter? En I. Steiner \& P. Wanner (Eds.), Migrants and Expats: The Swiss Migration and Mobility Nexus (pp. 
189-220). Springer International Publishing. https://doi.org/10.1007/9783-030-05671-1_8

Betts, A., \& Kainz, L. (2017). The history of global migration governance (Vol. 122). https://www.rsc.ox.ac.uk/publications/the-history-of-global-migrationgovernance

Bogotá, Alcaldía Mayor. (2018). Circular 037 del 24 de diciembre de 2018. https:// secretariageneral.gov.co/sites/default/files/marco-legal/circular_37.pdf Bogotá, Alcaldía Mayor. (2019). Circular 012 del 2 de abril de 2019. https:// www.alcaldiabogota.gov.co/sisjur/normas/Norma1.jsp?i=83369

Bogotá, Secretaría Distrital de Integración Social, (2019). Derecho de petición.

Respuesta de solicitud No.1380332019.

Boswell, C. (2002). Addressing the causes of migratory and refugee movements: the role of the European Union. Working Paper, (73). https://www. unhcr.org/uk/3e03005fa.pdf

Butcher, K., \& Morrison, A. (1998). Cross-city evidence on the relationship between immigration and crime. Journal of Policy Analysis and Management, 17(3), 457-493.

Capron, G., \& Esquivel, M. (2016). El enclave urbano, lógica socioespacial de la periferia urbanizada y sus efectos sobre la segregación residencial y la fragmentación urbana. Cuadernos de Geografía: Revista Colombiana de Geografia, 25(2), 127-150. https://doi.org/10.15446/rcdg.v25n2.54720

Castles, S., De Haas, H., \& Miller, M. J. (2014). The age of migration: International population movements in the modern world. Macmillan International Higher Education.

Colombia, Ministerio de Relaciones Exteriores. (2003). Comisión Nacional Intersectorial de Migración.

Colombia, Ministerio de Relaciones Exteriores. (2011). Acta Comité TécnicoComité Intersectorial de Integración y Desarrollo Fronterizo.

Crépeau, F., \& Nakache, D. (2006). Controlling irregular migration in Canada-Reconciling security concerns with human rights protection. IRPP Choices, 12(1), 3-42.

Crépeau, F., Nakache, D., \& Atak, I. (2007). International migration: Security concerns and human rights standards. Transcultural Psychiatry, 44(3), 311-337. https://doi.org/10.1177/1363461507081634

Dietz, G. (2010). Frontier hybridisation or culture clash? Transnational migrant communities and sub-national identity politics in Andalusia, Spain. Journal of Ethnic and Migration Studies, 30(6), 1087-1112. https://doi. org/10.1080/1369183042000286269 
Duchacek, I. (1984). The international dimension of subnational self-government. Publius: The Journal of Federalism, 14(4), 5-31. https://doi.org/10.1093/ oxfordjournals.pubjof.a037513

Düvell, F. (2006). Irregular migration: a global, historical and economic perspective. En F. Düvell (Ed.), Illegal immigration in Europe: Beyond control? (pp. 1439). Palgrave Macmillan UK. https://doi.org/10.1057/9780230555020_2

El Tiempo. (16 de noviembre, 2018a). Así fue la primera noche en el albergue de los venezolanos. El Tiempo. https://www.eltiempo.com/bogota/llegadade-venezolanos-al-albergue-que-dispuso-el-distrito-293256

El Tiempo. (13 de noviembre, 2018b). Migrantes aceptaron reglas de convivencia en nuevo albergue. El Tiempo. https://www.eltiempo.com/bogota/nuevocampamento-para-venezolanos-en-medio-de-protestas-de-vecinos-293066

Estupiñán, K. (2018). Distrito adelantó la primera mesa de trabajo para atender migración venezolana. https://bogota.gov.co/en/node/21484

Faist, T. (2000). The volume and dynamics of international migration and transnational social spaces. Oxford Scholarship. https://doi.org/10.1093/acprof: oso/9780198293910.001.0001

Gill, E. R. (2016). National citizenship and civil marriage. In A. E. Cudd E W. Lee (Eds.), Citizenship and immigration-Borders, migration and political membership in a global age (pp. 17-31). Springer.

Giraudy, A., Moncada, E., E Snyder, R. (2019). Subnational research in comparative politics: Substantive, theoretical and methodological contributions. En Autores, Inside countries : subnational research in comparative politics (pp. 1-106). Cambridge University Press.

Global South Studies Center, University of Cologne. (2015). Concepts of the Global South-Voices from around the world. https://core.ac.uk/download/ pdf/33335548.pdf

Goodwin-Gill, G. (2007). Migrant rights and managed migration. En V. Chetail (Ed.), Globalisation, migration and human rights: international law under review (pp. 161-187). Bruylant.

Goodwin-Gill, G. (2008). Forced migration: Refugees, rights and security. In J. McAdam (Ed.), Forced migration, human rights and security (pp. 1-18). Jart Publishing.

Greenwood, M. J. (1985). Human migration: Theory, models, and empirical studies. Journal of Regional Science, 25(4), 521-544. https://doi. org/10.1111/j.1467-9787.1985.tb00321.x

Guarnizo, L. E. (2006). El Estado y la migración global colombiana. Red Internacional de Migración y Desarrollo, 6(1), 79-101. 
Guiraudon, V. (2002). European integration and migration policy: Vertical policy-making as venue shopping. Journal of Common Market Studies, 38(2), 251-271. https://doi.org/10.1111/1468-5965.00219

Hurrell, A. (2013). Narratives of emergence: Rising powers and the end of the Third World? Revista de Economía Politica, 33(2), 203-221.

Ibrahim, M. (2005). The securitization of migration: A racial discourse 1. International Migration, 43(5), 163-187. https://doi.org/10.1111/j.14682435.2005.00345.x

Karyotis, G. (2007). European migration policy in the aftermath of September 11. Journal Innovation: The European Journal of Social Science Research, 20(1), 1-17. https://doi.org/10.1080/13511610701197783

Keohane, R., \& Nye, J. (1977). Power and interdependence: World politics in transition. Little Brown Books.

Kolodziej, E. A. (Ed.). (2005). Security and international relations. https://doi. org/10.1017/CBO9780511614903

Kronsell, A. (2006). Methods for studying silences: gender analysis in institutions of hegemonic masculinity. En B. A. Ackerly, M. Stern, \& J. True (Eds.), Feminist methodologies for international relations. Cambridge University Press.

Lavenex, S. (2018). Failing forward' towards which Europe? Organized hypocrisy in the common European asylum system. Journal of Common Market Studies, 56(5), 1195-1212. https://doi.org/10.1111/jcms.12739

Lee, E. S. (1966). A theory of migration. Demography, 3(1), 47-57. https://doi. org/10.2307/2060063

Léonard, S. (2010). EU border security and migration into the European Union: Frontex and securitisation through practices. European Security, 19(2), 231254. https://doi.org/10.1080/09662839.2010.526937

Libian Barreto. (2018). Alcalde Peñalosa anuncia atención integral a venezolanos migrantes en Bogotá. https://bogota.gov.co/mi-ciudad/gobierno/venezolanosmigrantes-tendran-atencion-integral-por-parte-del-distrit

Morgenthau, H. (1949). Politics among nations the struggle for power and peace. Alfred Knopf.

Mouffe, C., \& Laclau, S. (2007). En torno a lo político. Fondo de Cultura Económica (FCE).

Murga-Menoyo, M., E Novo, M. (2017). Sostenibilidad, desarrollo "glocal" y ciudadanía planetaria. Referentes de una pedagogía para el desarrollo sostenible. Teoría Educacional, 29(1), 55-78. https://doi.org/10.14201/ teoredu20172915578 
Navarrete-Yáñez, B. (2017). Percepciones sobre inmigración en Chile. Migraciones Internacionales, 9(1), 180-210.

Newton, L. (2008). Illegal, alien, or immigrant: The politics of immigration reform. NYU Press.

Nye, J. (2010). American power on decline? Foreign Affairs. https://www. foreignaffairs.com/articles/2010-11-01/future-american-power

Organización de las Naciones Unidas. (2019). Migración. https://www.un.org/ es/sections/issues-depth/migration/index.html

Paquet, M. (2019). Subnational migration states and the new politics of immigration. International Migration, 58(6), 61-76. https://doi.org/10.1111/ imig. 12649

Paul, K. T., \& Haddad, C. (2019). Beyond evidence versus truthiness: toward a symmetrical approach to knowledge and ignorance in policy studies. Policy Sciences, 52(2), 299-314. https://doi.org/10.1007/s11077-019-09352-4 Prieto Rosas, V., \& López Gay, A. (2015). Push and pull factors of Latin American migration. En A. Domingo, A. Sabater, \& R. R. Verdugo (Eds.), Demographic Analysis of Latin American Immigrants in Spain: From Boom to Bust (pp. 1-27). Springer International Publishing. https://doi.org/10.1007/9783-319-12361-5_1

Proyecto Migración Venezuela. (2019). En Colombia ya hay más de 1.4 millones de venezolanos. https://migravenezuela.com/web/articulo/cuantos-venezolanos-hay-en-colombia-hasta-hoy/1390

Schiavon, J. (2015). Una década de acción internacional de los gobiernos locales mexicanos (2005-2015). Revista Mexicana de Política Exterior, (104), 103-127.

Secretaría Distrital de Integración Social. (2018). En el Centro Integral de Atención al Migrante (CIAM), ya se atendieron a 4.360 personas. https://www. integracionsocial.gov.co/index.php/noticias/116-otros/3201-en-el-centrointegral-de-atencion-al-migrante-ciam-ya-se-atendieron-a-4-360-personas

Semana. (18 de septiembre, 2018). La solidaridad de Bogotá con los venezolanos que llegan. https://www.semana.com/nacion/articulo/venezolanosen-bogota-como-es-el-plan-de-ayuda/570407/

Sharp, J. (2011). Subaltern geopolitics: introduction. Geoforum Subaltern Geopolitics: Special Issue of Geoforum, 42(3), 271-273.

Smith, M. P. (2017). Transnational ties: Cities, migrations, and identities. En M. P. Smith \& J. Eade (Eds.), Transnational ties: Cities, migration, and identities (pp. 3-13). Routledge.

Soysal, Y. N., \& Soyland, A. J. (1994). Limits of citizenship: Migrants and postnational membership in Europe. University of Chicago Press. 
Suárez-Cao, J., Batlle, M., \& Wills-Otero, L. (2017). El auge de los estudios sobre la política subnacional latinoamericana. Colombia Internacional, (90), 15-34. https://doi.org/10.7440/colombiaint90.2017.01

Tatar, A. M. (2019). Panorama migratorio entre España y Colombia. Papel Político, 24(2).

Tovar, H. (2006). Emigración y éxodo en la historia de Colombia. Amérique Latine Histoire et Mémoire. Les Cahiers ALHIM, 3. https://doi.org/10.4000/ alhim. 522

Zapata, A. (4 de enero, 2019). Venezolanos vuelven a quedarse sin albergue en Bogotá. El Tiempo. https://www.eltiempo.com/bogota/venezolanosvuelven-a-quedarse-sin-albergue-en-bogota-311116 